\title{
Grupos de terapia ocupacional em telessaúde na pandemia de Covid-19: perspectivas de um Hospital-Dia de Saúde Mental
}

\section{Occupational therapy telehealth groups in Covid-19 Pandemic: Perspectives from a Mental Health Day Hospital}

Sonia Maria Leonardi Ferrari ${ }^{\mathrm{a}}$ (D), Samantha Domenique Pywell ${ }^{\mathrm{b}}$ (D), Ana Lucia Borges da Costa $^{\mathrm{b}}$ (D), Taís Quevedo Marcolino ${ }^{c}$ (D)

anstituto "A CASA", São Paulo, SP, Brasil.

'University of Central Lancashire, Preston, United Kingdom.

'Universidade Federal de São Carlos - UFSCar, São Carlos, SP, Brasil.

Como citar: Ferrari, S. M. L., Pywell, S. D., Costa, A. L. B., \& Marcolino T. Q. (2022). Grupos de terapia ocupacional em telessaúde na pandemia de Covid-19: perspectivas de um Hospital-Dia de Saúde Mental. Cadernos Brasileiros de Terapia Ocupacional, 30, e3019. https://doi.org/10.1590/2526-

8910.ctoRE22883019

\begin{abstract}
$\underline{\text { Resumo }}$
A pandemia de COVID-19 teve sério impacto ocupacional em pessoas com transtornos mentais pré-existentes. Para prestar o cuidado nesse contexto, os grupos de telessaúde foram uma opção terapêutica para terapeutas ocupacionais para o cuidado em saúde mental. Este artigo apresenta uma experiência de terapia ocupacional com grupos de telessaúde no Brasil, sustentada pelo Método Terapia Ocupacional Dinâmica, buscando discutir o uso da tecnologia durante a pandemia de Covid-19, além dos limites e das potencialidades do cuidado em terapia ocupacional diante da necessária mudança do trabalho com grupos. Trata-se de análise crítica da prática sustentada por uma perspectiva de produção de evidências baseadas na prática por meio de uma parceria colaborativa entre profissionais e acadêmicos do Brasil e do Reino Unido. Os profissionais refletiram sobre as habilidades necessárias no contexto online, destacando suas preocupaçóes iniciais e suas descobertas neste novo cenário de prática. A prática de cuidado em grupo de terapia ocupacional em telessaúde na prática de saúde mental requer o uso de múltiplas ferramentas digitais. Além disso, a(o) terapeuta ocupacional precisa entender das questóes de desigualdade digital (acesso digital e/ou habilidades digitais), aprimorar-se digitalmente para atender às necessidades das pessoas sob seu acompanhamento, além de possuir referenciais teórico-metodológicos claros que permitam sustentar práticas em telessaúde.
\end{abstract}

Palavras-chave: Terapia Ocupacional, Serviços de Atendimento, Terapia de Grupo, Surto do Novo Coronavírus 2019. 


\begin{abstract}
$\underline{\text { Abstract }}$
COVID-19 pandemic had a serious occupational impact on people with preexisting mental disorders. To deliver care in this context, telehealth groups were a therapeutic option for occupational therapists for mental health care. This paper presents an occupational therapy experience with telehealth groups in Brazil, sustained by the Dynamic Occupational Therapy Method, seeking to discuss the use of technology during the COVID-19 outbreak, the limits and potential of occupational therapy in the face of the necessary change in work with groups. The paper is a critical analysis of practice sustained by a practice-based evidence perspective through a collaborative partnership between practitioners and academics from Brazil and the UK. Practitioners reflected on their professional skills in an online context, highlighting their initial concerns and their discoveries within this new practice scenario. The delivery of telehealth groupwork in occupational therapy in mental health practice requires multiple digital tools, and the occupational therapist needs to understand digital inequity issues (digital access or skills), be digitally upskilled to meet client needs, and also be guided by clear occupational therapy theoretical and methodological frameworks that underpin telehealth practices.
\end{abstract}

Keywords: Occupational Therapy, Answering Service, Group Therapy, 2019 Novel Coronavirus Outbreak.

\title{
Introduçáo
}

O advento da pandemia de COVID-19 exigiu distanciamento social para enfrentála e nos colocou em uma situação de diversas ameaças às ocupaçôes. $\mathrm{O}$ isolamento compromete o ritmo social, alterando a rotina e gerando o aumento do estresse, medo e ansiedade como usual em situaçôes de interrupção ocupacional (Hammell, 2020). Em pessoas com transtornos mentais pré-existentes, todos esses problemas podem surgir com maior gravidade, com possíveis prejuízos nas relaçóes sociais, nas atividades de autocuidado e na adesão à medicação (Chatterjee et al., 2020; Usher et al., 2020; World Federation of Occupational Therapists, 2020).

Como Hammell (2020) argumenta, terapeutas ocupacionais lidam com processos de reconstrução de vida após interrupçóes ocupacionais. Nesse processo, destaca-se a importância de aprender a cuidar de si mesmo e dos outros, fazer escolhas e vivenciar sentimentos de pertencimento e conexão, prazer, propósito e significado ao se engajar em ocupaçóes. Pessoas com transtornos mentais se beneficiam de cuidados que as ajudem a organizar sua rotina, gerenciar o tempo e viver experiências de prazer e satisfação com o fazer, ajudando-as a construir um senso positivo de autoestima e esperança (Hammell, 2020; Mello et al., 2021).

Enfrentando os desafios de oferecer atendimento durante a pandemia, terapeutas ocupacionais rapidamente precisaram incorporar abordagens e metodologias emergentes, como o atendimento por telessaúde, mantendo valores centrados na pessoa e baseados na ocupação (Scott, 2020). A telessaúde se caracteriza pelas tecnologias de informação e comunicação na área da saúde quando clientes e profissionais se encontram 
em locais físicos distintos. As interaçóes por meio digital podem ocorrer em tempo real, de forma síncrona, como em uma ligação telefônica, por videoconferência ou por aplicativos (apps). As trocas também podem ocorrer de forma assíncrona quando as informações são armazenadas e encaminhadas por e-mail, vídeos, fotos, áudios (World Federation of Occupational Therapists, 2014).

$\mathrm{Na}$ terapia ocupacional, os recursos de telessaúde podem ser utilizados nas diversas etapas do processo terapêutico, incluindo o trabalho em grupo, respeitando as normas de prática de cada país (World Federation of Occupational Therapists, 2014). O trabalho em grupo é uma das estratégias de cuidado que impulsiona o profissional a encontrar soluçóes para sua oferta, sendo a videoconferência uma possibilidade. Há um consenso na literatura de terapia ocupacional de que o trabalho em grupo é um componente central dentro do conjunto de habilidades da profissão. Duncan (2011) descreve o trabalho em grupo dentro do conjunto de habilidades básicas para terapeutas ocupacionais. O trabalho em grupo tradicional, que ocorre presencialmente, pode ser feito com uma ampla variedade de possibilidades.

No entanto, devido à pandemia de COVID-19 e ao distanciamento social, as complexidades do trabalho em grupo passaram a incluir diferentes camadas envolvendo a equipe, os usuários dos serviços e a família. Terapeutas ocupacionais precisam reconhecer e trabalhar suas habilidades digitais para favorecer as pessoas com as quais trabalham para que realizem atividades e ocupaçóes (Hoel et al., 2021; Proffitt et al., 2021). Além disso, como alerta Pitliuk (2020), é necessário ter referências teóricas e metodológicas claras para embasar nossas intervençóes, especialmente neste cenário. A complexidade do ambiente virtual e a experiência e confiança no uso da tecnologia precisam ser sustentadas por uma estrutura teórico-metodológica que ajude a conduzir os processos terapêuticos.

Embora terapeutas ocupacionais possam possuir habilidades digitais, tecnologia e acesso para a oferta do trabalho em grupo online, as pessoas com as quais trabalham podem não ter acesso a um computador, tablet ou telefone celular. Presumir que os clientes tenham acesso à tecnologia necessária e possam participar do trabalho em grupo é uma falácia. A cisão digital, alfabetização digital e questóes éticas, portanto, precisam ser consideradas no raciocínio profissional antes de sua aplicação (Butler et al., 2008; Almathami et al., 2020). Apenas recomendar o trabalho em grupo online para os usuários dos serviços não é suficiente, e pode até se tornar uma barreira para o cuidado (Butler et al., 2008; Almathami et al., 2020). Terapeutas ocupacionais devem considerar quais são as circunstâncias desse indivíduo e suas necessidades para acessar o trabalho em grupo online antes de indicá-lo (Hoel et al., 2021; Proffitt et al., 2021).

Butler et al. (2008) dão destaque para a responsabilidade de liderança do grupo, que pode ser atribuída também aos terapeutas ocupacionais. Esses autores discutem que os grupos online requerem constante atenção, pois é mais fácil para um participante sair de um grupo online do que um grupo presencial. Terapeutas ocupacionais, portanto, precisam estar atentas(os) às suas responsabilidades de liderança no contexto online para a gestâo tecnológica e social, como sugerem Butler et al. (2008).

Nem todos vão rapidamente reconhecer que o mundo mudou da noite para o dia. Usuários dos serviços, familiares e mesmo profissionais da equipe podem não ser capazes de entender toda a transformação. Eles podem não ser capazes de assimilar o conceito de que um serviço que funcionava presencialmente passou a funcionar a distância, pois 
eles podem não conectar a situação atual com o que estavam acostumados. Alguns dos benefícios para os grupos online abarcam economia de custos com transporte, privacidade de fazer o grupo em sua casa (caso o ambiente doméstico permita), o que pode passar a ser uma preferência da pessoa. Reconhecer os prós e os contras permitirá que terapeutas ocupacionais ouçam ativamente as circunstâncias da pessoa em atendimento e explorem quais ajustes são necessários.

Embora terapeutas ocupacionais estejam enfrentando esses desafios, há uma chamada para avançar no conhecimento sobre a aplicação baseada em evidências em telessaúde, com ênfase no uso da tecnologia para avaliar e intervir no contexto da vida cotidiana e na própria oferta da telessaúde (Proffitt et al., 2021). A investigação colaborativa entre profissionais e pesquisadores se apresenta como uma estratégia fértil para ampliar as evidências baseadas na prática (Gélinas, 2016) de terapia ocupacional em telessaúde. Este artigo, por meio de uma análise situada de uma prática desenvolvida no Brasil, busca contribuir para a identificação de pistas positivas nessa temática.

Em São Paulo, Brasil, terapeutas ocupacionais tiveram que lidar rapidamente com o novo contexto da pandemia para fornecer um tratamento responsável e apropriado diante das circunstâncias únicas de restriçóes sociais (Malfitano et al., 2020; World Federation of Occupational Therapists, 2020). As normas regulatórias para a terapia ocupacional em telessaúde no Brasil foram rapidamente implementadas, garantindo que o trabalho das(os) terapeutas ocupacionais pudesse ser oferecido a diversos grupos populacionais (Ricci et al., 2020). Não houve política de lockdown no Brasil para enfrentar a pandemia (The Lancet, 2020). Mesmo assim, muitos serviços de saúde brasileiros incorporaram práticas de telessaúde, em busca de recursos de rastreamento, atenção e tratamento a distância, com benefícios à saúde, mesmo indiretamente relacionados ao COVID-19 (Gois-Santos et al., 2020; Caetano et al., 2020).

Em decisão conjunta entre equipe, usuários do serviço e familiares, um hospital-dia de saúde mental, na cidade de São Paulo, seguindo as diretrizes da OMS (World Health Organization, 2020), decidiu migrar para o tratamento de telessaúde para prevenir a disseminação do vírus. A responsabilidade pela continuidade do tratamento dos usuários levou o hospital-dia a oferecer grupos de telessaúde (Associação Brasileira dos Terapeutas Ocupacionais et al., 2020; World Federation of Occupational Therapists, 2014). Grupos de telessaúde foram usados como uma opção terapêutica na pandemia (Hoel et al., 2021; Pavani et al., 2021; Silva et al., 2020), mas as teorias de grupo existentes não foram desenvolvidas para telessaúde. Para contribuir com a literatura da terapia ocupacional ao discutir as complexidades da pandemia, da tecnologia e do potencial das(os) terapeutas ocupacionais para mudar a forma como oferecem terapia ocupacional em grupo, este artigo apresenta uma análise da prática de grupos de telessaúde de terapia ocupacional no contexto brasileiro.

\section{Método}

Este artigo se caracteriza como uma reflexão crítica sobre a prática. Em busca de evidências baseadas na prática (Gélinas, 2016), uma terapeuta ocupacional expert, brasileira, com 45 anos de experiência em saúde mental, e três acadêmicas, uma delas do Brasil e outras duas do Reino Unido, desenvolveram uma parceria colaborativa. 
Em maio de 2020, as acadêmicas estavam envolvidas em discussóes sobre como enfrentar os desafios impostos pela pandemia para realizar atividades de estágio no Brasil e no Reino Unido. Na universidade brasileira, estava ocorrendo uma experiência de estágio remoto em um cenário de prática de saúde mental, supervisionado pela terapeuta ocupacional experiente, a primeira autora do artigo. A primeira interaçáo entre as acadêmicas e a profissional ocorreu em uma atividade de intercâmbio educacional em outubro de 2020, quando a terapeuta ocupacional apresentou sua experiência com grupos de telessaúde em um hospital-dia de saúde mental para as estudantes do Reino Unido.

Dadas as excelentes discussóes instigadas pelo compartilhamento dessa experiência prática, as autoras decidiram analisar e avaliar mais profundamente os limites e os pontos fortes do trabalho em grupo de telessaúde como uma abordagem de tratamento nova, em evolução e flexível. Os procedimentos metodológicos para esta análise de prática incluíram: (a) a descrição da experiência na primeira pessoa; (b) a identificação de questóes relevantes a serem aprofundadas em um diálogo com a literatura de campo; e (c) a seleção de questóes decorrentes desta experiência prática para pesquisas futuras.

\section{Aspectos éticos}

A ênfase do artigo está na prática da terapeuta ocupacional e não nos detalhes da experiência particular dos participantes do grupo. Os aspectos éticos para relatos de experiência foram seguidos. Há confidencialidade sobre a identidade dos usuários do serviço, bem como alteraçóes em dados confidenciais sensíveis que permitem a identificação.

\section{Contexto da prática}

A prática em análise neste artigo aconteceu em um hospital-dia de saúde mental na cidade de São Paulo, no Brasil, um serviço privado de saúde com cobertura de diversos convênios de saúde, o qual oferece atendimento multidisciplinar para pessoas com sofrimento psíquico, sem vulnerabilidade econômica. O hospital-dia é organizado em torno de grupos terapêuticos, como psicoterapia, terapia ocupacional, culinária, assembleia, rádio, teatro, passeios e iniciativas de geração de renda (Ferrari, 2015).

A experiência aqui relatada é sustentada pelo Método Terapia Ocupacional Dinâmica (MTOD), desenvolvido por Jô Benetton no Brasil desde a década de 1970 (Marcolino et al., 2020). O objetivo principal do MTOD é a ampliação dos espaços de saúde no cotidiano, buscando inserção e participação social. A palavra "sujeito-alvo" é usada no MTOD como semelhante ao termo "cliente" - que é menos comum na terapia ocupacional brasileira (Gomes et al., no prelo).

No MTOD, não trabalhamos com protocolos de avaliação inicial, mas sim com um diagnóstico situacional contínuo, que envolve conhecer o sujeito-alvo na sua forma de ser, fazer e de se relacionar. O objetivo é compreender as necessidades do sujeito-alvo situacionalmente, por meio de observação cuidadosa e investigativa no setting, na vida cotidiana e por meio de informaçóes do sujeito-alvo e de outras pessoas relevantes, como outros profissionais ou familiares (sempre obtidas com o consentimento da pessoa). 
Outros instrumentos de avaliação podem ser utilizados posteriormente, com base nas necessidades identificadas no diagnóstico situacional (Marcolino et al., 2020).

O processo de intervenção está centrado no movimento dinâmico da relação triádica (terapeuta ocupacional, sujeito-alvo e atividades), buscando o estabelecimento de uma relação positiva na qual afetos são mobilizados e manejados para que a pessoa descubra desejos, habilidades e limitaçóes, favorecendo o surgimento do "desejo de aprender, fazer, seguir em frente" (Marcolino et al., 2020, p. 1325). Trata-se um arcabouço teórico-metodológico flexível, no qual as atividades são consideradas mais um termo da relação triádica, tão importante quanto $\mathrm{a}(\mathrm{o})$ terapeuta ocupacional e o sujeito-alvo, principalmente porque dão maior dinamismo à relação e permitem novas ocorrências, que podem posteriormente ganhar novos significados, em um processo reflexivo de construção de sentidos (Mello et al., 2020).

No ambiente grupal, a presença de outros participantes amplia as possibilidades de novas ocorrências, o que adiciona uma nova camada de complexidade e dinamismo à intervenção. As atividades são desenvolvidas por meio dos interesses, desejos e necessidades dos participantes, imprimindo ao grupo dois tipos de funcionamento: como atividade grupal (todos os participantes fazem a mesma atividade) ou grupo de atividades (cada um, no mesmo ambiente do grupo, faz sua própria atividade) (Ferrari, 2015).

Os resultados serão apresentados por meio de um texto descritivo na primeira pessoa, seguido de uma discussão com a literatura da área desencadeada por pontos selecionados da experiência prática.

\section{Resultados e Discussão}

Nosso tempo para preparar e criar alternativas para nossas intervençóes foi curto. Interrompemos os grupos presenciais no dia 15 de março de 2020, mantendo apenas um turno diário para emergências. Paralelamente, cada equipe organizou seus pacientes num aplicativo de mensagens e deu informaçóes sobre a plataforma online escolhida para celular e PC. O grupo de telessaúde de terapia ocupacional, foco desta análise prática, teve início no dia 23 de março, ministrado por: duas terapeutas ocupacionais seniores, que dividiam a coordenação do grupo; um terapeuta ocupacional recémformado; uma estagiária; e 12 usuários do serviço. Cada sessão de grupo durou um hora e meia, duas vezes por semana.

A rotina de nossos clientes era construída em torno do deslocamento diário para o hospital-dia. Quando essa rotina foi interrompida por causa da pandemia, ocorreu uma desorganização significativa da vida cotidiana. Assim, nosso raciocínio clínico precisava focar em encontrar oportunidades para ajudar os sujeitos-alvo em seu dia a dia, mantendo sua rotina e/ou criando alternativas. Compreendemos também que esse contexto se apresentava como uma oportunidade única de melhorar a situaçáo de cada um, compreender melhor quem eram, como estavam vivendo e quais eram suas necessidades nessa situação.

No início, havia apenas dúvidas: o ambiente online seria adequado e apropriado para as pessoas que atendemos? Como as sessóes aconteceriam sem a concretude dos materiais e ferramentas? E, acima de tudo, como se dariam sem a presença física, uma necessidade essencial para a maioria delas? Como se daria essa experiência de cuidar estando cada 
um de nós em nossas casas? São muitas as reflexões em torno dos desafios e das barreiras que precisam ser enfrentadas para a oferta equânime desse tipo de atendimento (Almathami et al., 2020).

No início, alguns clientes estavam mais familiarizados com a plataforma. Outros tiveram muitas dificuldades que julgávamos intransponíveis, como não ter celular nem acesso à internet. Aos poucos, encontramos soluçôes e os usuários superaram muitas dificuldades com nossa ajuda e com a ajuda de familiares e amigos. Iniciamos com atendimentos individualizados com os que tiveram maior dificuldade em aprender a usar a plataforma e contamos com o apoio dos familiares.

Os grupos, inicialmente, estavam voltados para o uso da plataforma. Tivemos que ensiná-los a ligar e desligar o som e a usar os fones de ouvido. Nós os ajudamos a localizar o melhor acesso à internet e os orientamos sobre onde colocar seus dispositivos para que pudessem nos ver melhor. Também ensinamos como mudar seus planos de fundo durante uma videochamada para garantir privacidade e oferecer opçóes. Surpreendentemente, os grupos começaram a trabalhar de forma muito criativa! A ideia de uma atividade grupal - em que todos fazem a mesma atividade - pareceu bem-vinda para esse novo ambiente. No início, sugerimos um jogo, o que não funcionou bem. Porém, um participante nos deu a ideia de fazer origami e todos aceitaram. Foi um sucesso! O participante ensinou a todos o passo a passo para fazer um "Tsuru”. Depois disso, fizemos uma atividade chamada $A$ vista da minha janela durante a quarentena, na qual os participantes descreveram fatos interessantes envolvendo a visão de sua janela. Esse grupo já se conhecia, o que tornou essa atividade possível. É preciso levar em consideração que as pessoas podem não querer mostrar o ambiente em que vivem, devido às condiçóes decorrentes das desigualdades sociais e da exclusão digital (Butler et al., 2008; Almathami et al., 2020).

Após essas primeiras atividades grupais, fizemos alguns exercícios de alongamento, exercícios de relaxamento e visitas virtuais a museus. Também viajamos ao redor do mundo usando um aplicativo que simula um passeio de carro por qualquer local do mundo e permite sintonizar uma estação de rádio em tempo real, no idioma daquele país. A gama de atividades grupais começou a aumentar: compartilhar na tela e contar sobre suas fotos favoritas; escolher e compartilhar os videoclipes das bandas favoritas, assim como seus artistas e cançóes favoritas; trocar receitas; desenvolver um artigo para a revista do hospital-dia; fazer uma colagem em grupo usando um aplicativo de design gráfico. Para que todas essas atividades se tornassem realidade, a terapeuta ocupacional teve que ganhar expertise para gerenciar todos os aplicativos, oferecendo flexibilidade para o trabalho online de grupo, mas principalmente permitindo que os participantes aprendessem e desenvolvessem suas necessidades singulares superando as dificuldades no uso das tecnologias (Almathami et al., 2020).

Normalmente, no trabalho em grupo, a(o) terapeuta ocupacional precisa estar atenta(o) à dinâmica do grupo e às necessidades de cada cliente (Finlay, 1997). No ambiente online, há outra camada complexa: a atenção para a gestão "tecnológica" e "social” (Butler et al., 2008). É uma demanda maior de atenção e observação para monitorar os movimentos de cada janela: quem saiu? Quem reagiu? Existem pistas de comunicação muito particulares nesse contexto, e o grau de proximidade e percepção do outro pode ser ainda mais intenso. Certa vez, ocorreu uma situação em que um participante tinha certeza de que todos estavam olhando apenas para ele e saiu da 
reunião. Durante o tempo todo, a $(\mathrm{o})$ terapeuta precisa observar, perguntar para entender os motivos do que foi observado e intervir explicando como funciona o ambiente virtual.

As intervençóes se centraram nas necessidades de cada cliente, oriundas do trabalho em grupo, como: encontrar soluçóes criativas para uma participante tomar um banho de modo mais privado enquanto estava na casa da mãe; ajudar outro participante a ir ao banco para pegar o dinheiro do auxílio emergencial; ajudar outra participante a se organizar para uma cirurgia, acompanhando-a virtualmente até a consulta, e, no dia da cirurgia, acompanhando-a até o momento em que ela entrou na sala de operação. $\mathrm{O}$ aplicativo de mensagens também se tornou uma extensão do grupo, possibilitando intervençóes assíncronas, como: conversar sobre o que estavam vivenciando, ajudar uns aos outros e compartilhar ideias relacionadas às atividades que eles poderiam fazer no próximo encontro. A composição do uso de diferentes tecnologias possibilitou ampliar as possibilidades de interação entre os participantes. Esse cenário ampliado permitiu a vivência de situações às quais não teríamos oportunidade de viver se não fosse o ambiente virtual, mostrando-nos possibilidades de ampliação de relaçóes e atividades que podem permanecer híbridas no futuro.

Assim como em nossa abordagem presencial, continuamos facilitando atividades individuais ou coletivas. Alguns participantes tiveram acesso a recursos materiais em casa e puderam desenvolver atividades durante as sessóes de grupo (por exemplo, pinturas, trabalhos manuais). Outros tiveram suas atividades relacionadas a sua nova rotina. $\mathrm{O}$ grupo acabou apoiando uns aos outros, apesar das diferenças significativas nas necessidades de saúde mental. Para alguns, o desespero de estar em casa era muito pior do que o medo do vírus. Para outros, o extremo conforto de não precisar enfrentar o mundo exterior resultou no desejo de não querer sair de casa. Apesar da literatura existente sobre o trabalho em grupo com foco em grupos presenciais, analisamos que os grupos de telessaúde também possibilitaram o estreitamento dos relacionamentos e a promoção da ajuda mútua, objetivos de todo trabalho em grupo (Finlay, 1997; Duncan, 2011).

Uma usuária, Ellen, sempre teve muitas dificuldades em participar de grupos face a face pois sempre se sentiu ameaçada pelos outros, participando com certa distância e em seu ritmo. No início, e em muitas reuniôes online, ela sempre participava com o áudio desligado. As pessoas do grupo a alertavam sobre essa condição, mas ela apenas ria e demonstrava que estava bem por meio de gestos. No entanto, ela sempre foi altamente conectada ao grupo. Durante uma conversa com sua irmá, ela nos informou que Ellen estava bem em casa. Ela disse que Ellen saiu do estado depressivo com a participação em grupos de telessaúde e voltou a ler, ouvir música e a se comunicar mais com a família.

Isso nos surpreendeu! Certo dia, Ellen começou a abrir seu microfone e atender a nossos pedidos. Ela gradualmente participou de algumas atividades. Outras vezes, Ellen prestava muita atenção, mas recusava o convite para participar. Por fim, ela participou de uma sessão na qual compartilhou um vídeo da Sinfonia para Violino de Bach que ela gosta muito. Essa foi uma atividade importante porque ela aprendeu a tocar violino em algum momento de sua vida. Já a havíamos convidado para trazer seu violino e tocar para nós, mas ela sempre recusou. Compartilhar a sinfonia foi o mais próximo que conseguimos chegar dessa atividade que lhe é singular.

Outro usuário, George, causou muita preocupação porque foi intensamente afetado pelo isolamento/confinamento e não conseguia se organizar em casa para tomar seus 
remédios, alimentar-se adequadamente ou usar o aplicativo de reunião em grupo. Em resposta a isso, oferecemos várias sessóes individuais para ajudá-lo nas tarefas diárias e para ensiná-lo a usar o aplicativo. Como resultado do atendimento ao grupo de telessaúde de terapia ocupacional, ele voltou a algumas de suas ocupaçóes e conseguiu retomar atividades de autocuidado, resultando em uma melhora significativa de seu humor e de seus processos de pensamento. Ele começou a gostar do espaço do grupo e aprendeu com as experiências dos demais, além de ampliar seus interesses. Finalmente, ele retomou o relacionamento com sua filha, de quem estava muito distante.

\section{Consideraçóes Finais}

Mais de um ano após o início da pandemia, esta nova prática ainda está em andamento. Daí nossa necessidade de compartilhar nossas reflexóes para analisar e avaliar os limites e as potencialidades do trabalho em grupo de telessaúde como uma abordagem de tratamento nova, em evolução e flexível. O trabalho em grupo de telessaúde de terapia ocupacional em ambientes de saúde mental tem muitos benefícios, especialmente durante a crise pandêmica; no entanto, investigaçóes mais aprofundadas sobre o impacto dessa forma de intervenção são necessárias.

A estrutura dinâmica do MTOD favoreceu esse novo desafio na medida em que nos permitiu oferecer uma intervenção à medida de cada um e, ao mesmo tempo, aberta a novas possibilidades mediadas pelas tecnologias. O processo de manejo de grupos de telessaúde possibilitou experimentar novas formas de fazer e estar no mundo. Também promoveu um sentimento de pertença, o que ajudou os nossos clientes a passar por esse momento difícil.

O trabalho em grupo de telessaúde na terapia ocupacional é possível, mas requer consideraçóes sobre a situação e as necessidades dos indivíduos (Almathami et al., 2020; Proffitt et al., 2021). O acesso à internet e à tecnologia e as habilidades digitais das pessoas em atendimento, familiares e até mesmo profissionais da equipe são os principais fatores para analisar a implementação de grupos de telessaúde (Hoel et al., 2021; Proffitt et al., 2021). É necessário investigar aspectos que caracterizam as boas práticas de trabalho em grupo em telessaúde e quais características influenciam o raciocínio profissional.

Como implicaçóes para a prática, destacamos que o trabalho em grupo de telessaúde em saúde mental demanda várias ferramentas digitais. Terapeutas ocupacionais precisam entender questóes da desigualdade digital (acesso e/ou habilidades digitais) e se aprimorarem digitalmente para atender às necessidades das pessoas em atendimento, além de utilizarem estruturas teórico-metodológicas de terapia ocupacional robustas que possam sustentar as práticas no contexto online. Estudantes de terapia ocupacional precisam aprender tais habilidades, a fim de se prepararem para trabalhos futuros. Além disso, embora as definiçóes de telessaúde incluam a oferta de grupos online, ainda há necessidade de estudos que melhor delineiem suas especificidades.

\section{Referências}

Almathami, H., Win, K. T., \& Vlahu-Gjorgievska, E. (2020). Barriers and facilitators that influence telemedicine-based, real-time, online consultation at patients' homes: systematic literature review. Journal of Medical Internet Research, 22(2), 1-25. http://dx.doi.org/10.2196/16407. 
Associação Brasileira de Terapeutas Ocupacionais - ABRATO, Silva, B. R., Oliveira, P. V. B., Folha, O. A. O. C., Nicolau, S. M., Wertheimer, L. G., Silva, D. R., Sousa, R. N. P., Pacheco, S. R. G., Krawczyk, N. S. O., Moraes, B. M., Omura, K. M., \& Marcolino, T. Q. (2020). Nota da Associaçáo Brasileira dos Terapeutas Ocupacionais - ABRATO sobre o COVID-19. RevisbraTO, 4(3), 281-289. Recuperado em 05 de janeiro de 2021, de https://revistas.ufrj.br/index.php/ribto/article/view/34390/pdf

Butler, B., Sproull, L., Kiesler, S., \& Kraut, R. (2008). Community effort in Online Groups: Who does the work and why? In S. P. Weisband (Org.), Leadership at a distance: research in technologicallysupported work (pp. 171-194). Oxford: Taylor and Francis Group.

Caetano, R., Silva, A. B., Guedes, A. C. C. M., Paiva, C. C. N., Ribeiro, G. R., Santos, D. L., \& Silva, R. M. (2020). Challenges and opportunities for telehealth during the COVID-19 pandemic: ideas on spaces and initiatives in the Brazilian context. Cadernos de Saúde Pública, 36(5), 1-16. https://doi.org/10.1590/0102-311x00088920.

Chatterjee, S. S., Barikar, C. M., \& Mukherjee, A. (2020). Impact of COVID-19 pandemic on preexisting mental health problems. Asian Journal of Psychiatry, 51, 1-2. https://doi.org/10.1016/j.ajp.2020.102071.

Duncan, E. (2011). Foundations for practice in occupational therapy. London: Churchill Livingstone.

Ferrari, S. M. L. (2015). Grupos de terapia ocupacional em saúde mental: novas reflexóes. In V. S. Maximino \& F. Liberman (Orgs.), Grupos e Terapia Ocupacional: Formação, pesquisa e açóes (pp. 226-237). São Paulo: Summus Editorial.

Finlay, L. (1997). Groupwork in occupational therapy. London: Nelson Thornes.

Gélinas, I. (2016). Partnership in research: a vehicle for reaching higher summits. Canadian Journal of Occupational Therapy, 83(4), 204-215. https://doi.org/10.1177/0008417416668859.

Gois-Santos, V. T., Freire, D. A., Libório, L. S., Ferreira, E. C., \& Santos, V. S. (2020). Telehealth actions in times of COVID-19: information with evidence. Revista da Associação Médica Brasileira, 66(10), 1320-1322. https://doi.org/10.1590/1806-9282.66.10.1320.

Gomes, L. D., Araújo, A. S., Cid, M. F. B., Ferigato, S. H., \& Marcolino, T. Q. (in press). Let's think about practice? The applicability of a reflexive tool to support professional reasoning in occupational therapy. Brazilian Journal of Occupational Therapy.

Hammell, K. W. (2020). Engagement in living during the COVID-19 pandemic and ensuing occupational disruption. Occupational Therapy Now, 22(4), 7-8.

Hoel, V., von Zweck, C., Ledgerd, R., \& World Federation of Occupational Therapists. (2021). Was a global pandemic needed to adopt the use of telehealth in occupational therapy? Work, 68(1), 13-20. https://doi.org/10.3233/WOR-205268.

Malfitano, A. P. S., Cruz, D. M. C., \& Lopes, R. E. (2020). Occupational therapy in times of pandemic: social security and guarantees of possible everyday life for all. Brazilian Journal of Occupational Therapy, 28(2), 401-404. https://doi.org/10.4322/2526-8910.ctoed22802.

Marcolino, T. Q., Benetton, J., Cestari, L. M. Q., Mello, A. C. C., \& Araújo, A. S. (2020). Dialogues with Benetton and Latour: possibilities for an understanding of social insertion. Brazilian Journal of Occupational Therapy, 28(4), 1322-1334. https://doi.org/10.4322/2526-8910.ctoarf2032.

Mello, A. C. C. M., Dituri, D. R., \& Marcolino, T. Q. (2020). The meaning making of what is meaningful: dialogues with Wilcock and Benetton. Brazilian Journal of Occupational Therapy, 28(1), 356-377. https://doi.org/10.4322/2526-8910.ctoen1896.

Mello, A. C. C., Araújo, A. S., Costa, A. L. B., \& Marcolino, T. Q. (2021). Meaning-making in occupational therapy intervention: a scoping review. Cadernos Brasileiros de Terapia Ocupacional, 29, 1-20. https://doi.org/10.1590/2526-8910.ctoAR2158.

Pavani, F. M., Silva, A. B., Olschowsky, A., Wetzel, C., Nunes, C. K., \& Souza, L. B. (2021). Covid-19 e as repercussóes na saúde mental: estudo de revisão narrativa de literatura. Revista Gaúcha de Enfermagem, 42(spe), 1-14. https://doi.org/10.1590/1983-1447.2021.20200188. 
Pitliuk, L. (2020). Sustentar uma clinica psicanalitica online? Instituto Sedes Sapientiae. Recuperado em 05 de janeiro de 2021, de https://youtu.be/rIY_yxWBiXk

Proffitt, R., Cason, J., Little, L., \& Pickett, K. A. (2021). Stimulating research to advance evidence-based applications of telehealth in occupational therapy. OTJR, 41(3), 153-162. https://doi.org/10.1177/15394492211011433.

Ricci, E. C., Dimov, T., Cassais, T. S., \& Dellbrügger, A. P. (2020). Occupational therapy in Brazil during the COVID-19 pandemic: peer support groups as mental health intervention strategy. World Federation of Occupational Therapists Bulletin, 77(1), 33-35. http://dx.doi.org/10.1080/14473828.2020.1840767.

Scott, J. (2020). Post Covid-19 in occupational therapy. British Journal of Occupational Therapy, 83(10), 607-608. https://doi.org/10.1177/0308022620957579.

Silva, M. A., Perez, O. F. R., Añez, L. M., \& Paris Junior, M. (2020). Telehealth treatment engagement with Latinx populations during the COVID-19 pandemic. The Lancet, 8(3), 176-178. https://doi.org/10.1016/S2215-0366(20)30419-3.

The Lancet. (2020). COVID-19 in Brazil: “So what?” The Lancet, 395(10235), 1461. https://doi.org/10.1016/S0140-6736(20)31095-3.

Usher, K., Bhullar, N., \& Jackson, D. (2020). Life in the pandemic: social isolation and mental health. Journal of Clinical Nursing, 29(15-16), 2756-2757. https://doi.org/10.1111/jocn.15290.

World Federation of Occupational Therapists - WFOT. (2014). Telehealth. Recuperado em 5 de janeiro de 2021, de https:/www.wfot.org/resources/telehealth

World Federation of Occupational Therapists - WFOT. (2020). Public Statement - Occupational Therapy Response to the COVID-19 Pandemic. Recuperado em 5 de janeiro de 2021, de https://www.wfot.org/about/public-statement-occupational-therapy-response-to-the-covid-19-pandemic

World Health Organization - WHO (2020). 2019 Novel Coronavirus (2019-nCov): Strategic preparedness and response plan. Geneva: WHO. Recuperado em 5 de janeiro de 2021, de https://www.who.int/publications/i/item/strategic-preparedness-and-response-plan-for-the-new-coronavirus

\section{Contribuiçáo dos Autores}

Todas as autoras contribuíram igualmente para o

manuscrito e aprovaram a versão final do texto.

\section{Autor para correspondência}

Sonia Maria Leonardi Ferrari

e-mail address: soferrari@gmail.com

\section{Editor de seçáo}

Prof. Dr. Milton Carlos Mariotti 